\title{
Linear scaling time-dependent density-functional tight-binding method for absorption spectra of large systems
}

\author{
Fan Wang, Chi Yung Yam, and GuanHua Chen* \\ Department of Chemistry, The University of Hong Kong, Pokfulam Road, Hong Kong \\ XiuJun Wang \\ Department of Chemistry, South China University of Technology, Guangzhou 510640, China \\ Kangnian Fan \\ Department of Chemistry, Fudan University, Shanghai 200433, China
}

Thomas A. Niehaus and Thomas Frauenheim ${ }^{\dagger}$

Bremen Center for Computational Materials Science, University of Bremen, D-28359 Bremen, Germany

(Received 14 February 2007; revised manuscript received 3 May 2007; published 24 July 2007)

\begin{abstract}
A time-dependent density-functional tight-binding method in real time domain is developed to calculate the absorption spectra of very large systems. The time-dependent first-order response of the density matrix due to an external perturbation is solved using Chebyshev method with high efficiency and accuracy. Linear scaling of CPU time and memory usage with the system size is achieved by exploring the sparsity of the involving matrices as well as by introduction of a cutoff for the first-order density matrix. The compressed sparse row scheme is used to store the matrices, and SPARSEKIT is employed for sparse matrix multiplication. The absorption spectra of three-dimensional water clusters $\left(\mathrm{H}_{2} \mathrm{O}\right)_{216},\left(\mathrm{H}_{2} \mathrm{O}\right)_{432},\left(\mathrm{H}_{2} \mathrm{O}\right)_{648}$, and $\left(\mathrm{H}_{2} \mathrm{O}\right)_{864}$ are calculated using the present approach. The error due to the cutoff of density matrix is negligible. It is shown from these calculations that the presented method is very efficient and capable of calculating the absorption spectra for very large three-dimensional systems.
\end{abstract}

DOI: 10.1103/PhysRevB.76.045114

PACS number(s): 31.10.+z, 31.15.Ew, 31.15.-p

\section{INTRODUCTION}

Time-dependent density-functional theory ${ }^{1-11}$ (TDDFT) shows great promise in the calculation of excitation energies at a relatively small computational effort. Within TDDFT, the excitation energies are decided by an eigenvalue equation. ${ }^{2,3}$

$$
\Omega F_{I}=\omega_{I}^{2} F_{I},
$$

where $\omega_{I}$ is the excitation energy. The dimension of matrix $\Omega$ is of $O\left(N^{2}\right)$, where $N$ is the number of the basis functions. The computational time to diagonalize this matrix scales as $O\left(N^{6}\right)$. In most cases, only transitions with low excitation energies are needed and iteration methods such as Lanczos method or Davidson's algorithm ${ }^{12}$ are thus adopted. In these iteration methods, the multiplications between $\Omega$ matrix and trial vectors are required and the excitation energies are decided through a diagonalization of a small matrix whose dimension is a little larger than the number of excitations to be decided. The multiplications are almost the same as the construction of Fock matrices in canonical molecular orbital basis. Usually, these multiplications are done first in atomic basis and then transformed to canonical molecular orbital basis. The iteration methods are very efficient in the determination of the lowest several excitation energies especially for small and medium sized systems. In addition, linear scaling algorithms ${ }^{13}$ can be applied to reduce the computational effort further in the calculation of the multiplications in the localized atomic orbitals, which is usually the most timeconsuming step. However, for very large systems, the itera- tion methods are not as efficient. First, the canonical molecular orbitals are usually delocalized and the transformation of a matrix from atomic basis to canonical molecular orbital basis scales as $O\left(N^{3}\right)$. Second, for very large systems, the number of excited states even in a small energy range could be huge, which makes the matrix diagonalization in the iteration methods a time-consuming task.

To solve this problem, the linear response of the density matrix due to an external field may be solved directly in real time domain, and the absorption spectra and dynamic polarizability in the whole energy range can thus be obtained through a Fourier transformation. ${ }^{14,15}$ In this approach, any type of basis functions can be adopted. The use of localized basis functions leads to the linear scaling of the computational effort with respect to the system size, and facilitates its application to large systems. In a previous work, ${ }^{15}$ we use Chebyshev approach ${ }^{16,17}$ to calculate the linear response of the density matrix to an external potential which is a timedomain $\delta$ function. This method has been shown to be able to calculate accurately the excitation energies of small systems with high efficiency. In addition, the linear scaling of computational efforts can be achieved through a cutoff of the density matrix in localized bases for large systems. This time-domain first-principles method has thus the potential to be applied to the calculation of absorption spectra and dynamics polarizability of large systems. ${ }^{14}$ However, with the current DFT implementation and computational power, it is still much too expensive to use the time-domain firstprinciples method to deal with large systems. This is because the construction of Fock matrix is very expensive for large 
systems, and the Fock matrix has to be constructed more than 1000 times in a normal calculation using this timedomain method. At the present moment, the time-domain method is therefore more suitable to be applied to large systems by being implemented at the semiempirical levels.

Over the last few years, a density-functional tight-binding, ${ }^{18-21}$ (DFTB) method has been developed for a wide range of applications. It has been used to calculate the low-lying singlet and triplet excitation energies based on Eq. (1) (Ref. 22) with a computational effort that is several orders of magnitude less than the traditional DFT methods. ${ }^{23}$ The time-dependent Kohn-Sham equations of DFTB have also been integrated in real time using a variant of the Cayley algorithm. ${ }^{24,25}$ This nonperturbative approach can be used to study molecular dynamics in the excited state as well as nonadiabatic effects but suffers from cubic scaling with system size. In the present work, the time-dependent DFTB method is developed to calculate the absorption spectra of large systems. We show that with a cutoff of the density matrix, the computational time scales linearly with the system size.

\section{THEORY}

\section{A. Time-domain TDDFT method}

Within TDDFT formalism, the equation of motion for the density matrix (in an orthogonal basis set) reads

$$
i \frac{d}{d t} P(t)=[h(t), P(t)]
$$

where $h(t)$ is the time-dependent Fock matrix, and $P(t)$ is the reduced single-electron density matrix. If the system is initially at its ground state and is perturbed by an external potential, the first-order change of density matrix follows the following equation:

$$
\begin{gathered}
i \frac{d}{d t} P^{(1)}(t)=\left[h_{0}, P^{(1)}(t)\right]+\left[h^{(1)}(t), P_{0}\right]+\left[V^{e x t}(t), P_{0}\right], \\
P^{(1)}(t=0)=0,
\end{gathered}
$$

where $P_{0}$ and $h_{0}$ are the ground-state density matrix and Fock matrix, respectively, $P^{(1)}$ is the first-order change of density matrix, $h^{(1)}$ is the first-order change of Fock matrix due to $P^{(1)}$, and $V^{e x t}(t)$ is the matrix of the external perturbation potential. When a time-dependent electric field is applied, the absorption spectra and dynamic polarizability can be obtained via the Fourier transformation of the timedependent first-order change of the dipole moment. ${ }^{14}$ The excitation energies can be determined from the absorption spectra. If the external perturbed potential is a $\delta$ function in time, Eq. (3) for $t>0$ becomes

$$
\begin{gathered}
i \frac{d}{d t} P^{(1)}(t)=\left[h_{0}, P^{(1)}(t)\right]+\left[h^{(1)}(t), P_{0}\right], \\
P^{(1)}\left(t=0_{+}\right)=-i\left[V^{e x t}, P_{0}\right] .
\end{gathered}
$$

Equation (4) is a set of linear differential equations since $h^{(1)}$ depends linearly on $P^{(1)}$ and can be written formally as

$$
i \frac{d}{d t} P^{(1)}=L P^{(1)}
$$

where $L$ is a time-independent linear Liouville operator. The formal solution for Eq. (5) is

$$
P^{(1)}(t)=e^{-i L t} P^{(1)}(t=0)
$$

The exponential operator $e^{-i L t}$ can be calculated through the Taylor expansion. However, the Taylor expansion is not efficient due to its power law convergence property. Alternatively the Chebyshev expansion for $e^{-i L t}$ can be employed, and has been shown to be more efficient. ${ }^{16,17}$ In the Chebyshev approach, $P^{(1)}(t)$ reads

$$
P^{(1)}(t)=e^{-i L t} P^{(1)}(t=0)=\sum_{n=0}^{\infty}\left(2-\delta_{n 0}\right) J_{n}(t \Delta) P_{n}
$$

$$
\begin{gathered}
P_{n}=\frac{-2 i}{\Delta} L P_{n-1}+P_{n-2}, \quad P_{1}=\frac{-i}{\Delta} L P_{n-1}, \\
P_{0}=P^{(1)} \quad(t=0),
\end{gathered}
$$

where $J_{n}(\alpha)$ is Bessel function of the first kind and $\Delta$ is a positive number larger than the largest eigenvalue of operator $L, L_{\max }$. The expansion converges for any time step $t$ as long as $\Delta$ is larger than $L_{\max }$. In actual calculations, $\Delta$ is estimated through the difference between the highest virtual level and the lowest occupied level. The expansion in Eq. (7) converges exponentially due to the exponential decay of the Bessel function $J_{n}(\alpha)$ when $n$ is larger than $\alpha$. The number of terms needed in Eq. (7) should only be slightly larger than $t \Delta$. To achieve high efficiency, a large time step is preferred. The first-order time-dependent properties at any intermediate time can be determined through Eq. (7) with little additional computational effort. This is done by saving the corresponding properties due to the calculated $P_{n}$. In a normal calculation, a propagation time of 35 fs results in an energy resolution of $0.1 \mathrm{eV}$ for the absorption spectra, which is usually accurate enough for large systems.

\section{B. DFTB and time-dependent DFTB}

In DFTB, the minimum Slater-type orbitals for valence electrons, which are determined by solving the selfconsistent field equation based on local density approximation $^{26,27}$ for free neutral atoms, are adopted as the basis functions. The exchange-correlation energy is expanded to the second order in the change of the electron density (with respect to a reference density $n_{0}$ ). This reference density is usually taken as the summation of the electronic densities of component atoms. The total energy is then written as ${ }^{18,19}$ 


$$
\begin{aligned}
E_{t o t}= & \sum_{i=1}^{o c c}\left\langle\psi_{i}\left|\hat{H}_{0}\right| \psi_{i}\right\rangle \\
& +\frac{1}{2} \iint\left[\frac{1}{\left|\vec{r}-\vec{r}^{\prime}\right|}+\left.\frac{\delta^{2} E_{X C}}{\delta n(\vec{r}) \delta n\left(\vec{r}^{\prime}\right)}\right|_{n=n_{0}}\right] \\
& \times \delta n(\vec{r}) \delta n\left(\vec{r}^{\prime}\right) d \vec{r} d \vec{r}^{\prime}-\frac{1}{2} \iint \frac{n_{0}(\vec{r}) n_{0}\left(\vec{r}^{\prime}\right)}{\left|\vec{r}-\vec{r}^{\prime}\right|} d \vec{r} d \vec{r}^{\prime} \\
& +E_{X C}\left[n_{0}\right]-\int V_{X C}\left[n_{0}, \vec{r}\right] n_{0}(\vec{r}) d \vec{r},
\end{aligned}
$$

where $\hat{H}_{0}$ is the Hamiltonian of the system with the reference electron density $n_{0}$, and $\delta n$ is the change of the electron density. It can be seen from Eq. (9) that the last three terms on the right hand side (rhs) of Eq. (9) do not depend on $\delta n$ or the density matrix of the system. These terms are evaluated as a sum of interaction of interatomic two-body potentials within DFTB. The $\hat{H}_{0}$ matrix is calculated based on a twocenter approximation and the matrix elements are tabulated together with the overlap matrix elements with respect to the interatomic distance. The second term on the rhs of Eq. (9) is further approximated by the interaction between the Mulliken charges $\Delta q_{a}$ on each atom as

$$
E^{(2)}=\frac{1}{2} \sum_{a, b} \gamma_{a b} \Delta q_{a} \Delta q_{b},
$$

where $\gamma_{a b}$ are parameters. The Fock matrix in DFTB can be obtained through the derivative of the total energy with respect to the density matrix as

$$
F_{\mu \nu}=\left\langle\chi_{\mu}\left|\hat{H}^{0}\right| \chi_{\nu}\right\rangle+\frac{1}{2} S_{\mu \nu} \sum_{\varsigma}\left(\gamma_{\alpha \varsigma}+\gamma_{\beta \varsigma}\right) \Delta q_{\varsigma},
$$

where $S_{\mu \nu}$ is the overlap matrix, and bases $\mu$ and $\nu$ are on atoms $\alpha$ and $\beta$, respectively.

The time-dependent DFTB (Ref. 22) (TD-DFTB) has been used to calculate the excitation energies. In Ref. 22, TD-DFTB was formulated in terms of Eq. (1), and the excitation energies of some small and medium sized systems were calculated with an accuracy close to approximated TDDFT. $^{23,28,29}$ TD-DFTB can also be formulated in terms of Eq. (4), and can be integrated in real time, which is expected to be applicable to very large systems. The first-order change of Fock matrix due to the density matrix is expressed as

$$
h_{\mu \nu}^{(1)}=\frac{1}{2} S_{\mu \nu} \sum_{\varsigma}\left(\gamma_{\alpha \varsigma}+\gamma_{\beta \varsigma}\right) \Delta q_{\varsigma}^{(1)},
$$

where $\Delta q_{\mathrm{s}}^{(1)}$ is the Mulliken charge on atom $\zeta$ due to the first-order change of density matrix:

$$
\Delta q_{\varsigma}^{(1)}=\sum_{\mu \in \varsigma} \sum_{\nu} P_{\mu \nu}^{(1)} S_{\mu \nu} .
$$

In TD-DFTB, the calculation of $h^{(1)}$ is much faster than the calculation of the commutators in Eq. (4) even for medium sized systems. For large systems, the sparsity of the matrices involved must be explored. It can be seen from Eqs. (11) and (12) that $h_{0}$ and $h^{(1)}$ are sparse matrices and the number of nonzero elements depends linearly on the system size. For systems with a finite highest occupied molecular orbitallowest unoccupited molecular orbital gap, the ground state density matrix $P_{0}$ is also a sparse matrix. ${ }^{30}$ The computational time for the commutator between $h^{(1)}$ and $P_{0}$ scales thus linearly with system size. The linear scaling of computational time can be achieved by introducing further a cutoff for $P^{(1)}$.

\section{COMPUTATIONAL DETAILS AND RESULTS}

The time-domain Chebyshev expansion of TD-DFTB based on Eq. (4) has been implemented in the LODESTAR program package. ${ }^{14,31,32}$ Equation (4) is formulated in an orthogonal basis and the Cholesky decomposition ${ }^{33}$ for the overlap matrix is used to orthogonalize the basis functions. The Cholesky decomposition has been shown to scale linearly with the system size due to the sparsity of the overlap matrix. ${ }^{14}$ In addition, the orthogonalized basis functions obtained in this way are still localized and the sparsity of the related matrices can be kept. Before the time propagation, the ground state Fock matrix and density matrix as well as the dipole moment matrices are transformed from the atomic orbital basis set to this orthogonal basis set. To perform the action of $L$ operator on $P^{(1)}$, four steps are taken: (1) to transform $P^{(1)}$ from the orthogonal orbital basis to the atomic orbital basis, (2) to calculate $h^{(1)}$ in the atomic orbital basis using Eqs. (12) and (13) based on $P^{(1)}$ in the atomic orbitals, (3) to transform $h^{(1)}$ from the atomic orbital basis set to the orthogonal basis set, and (4) to calculate the commutators in Eq. (4). Step 2 is actually the fastest step for large systems and the bottleneck is step 4. Furthermore, step 3 is faster than step 1 since $h^{(1)}$ is sparse while $P^{(1)}$ is generally not. However, this may not always be true if a cutoff for $P^{(1)}$ is applied. In addition, the computational effort of step 1 can be reduced using the fact that only those $P_{\mu \nu}^{(1)}$ elements in the atomic orbitals with corresponding nonzero $S_{\mu \nu}$ are needed since only these density matrix elements contribute to the Mulliken charge in Eq. (13). Among these steps, the computational efforts for steps 2 and 3 scale linearly with the system size, while the computational times of the other two steps scale as $O\left(N^{2}\right)$ if no cutoff is adopted for $P^{(1)}$.

The computational effort to calculate $P^{(1)}(t)$ via Eq. (7) is determined by the number of expansion terms in Eq. (7), which is in turn determined by $t \Delta$. In most TD-DFTB calculations, a $\Delta$ of $80 \mathrm{fs}^{-1}$ is usually large enough to ensure the convergence of the expansion in Eq. (7). As a result, the rhs of Eq. (4) needs to be calculated 3000 times for a $35 \mathrm{fs}$ propagation. An additional reduction in computational effort can be achieved by using the fact that the Fock matrix in DFTB is real and only depends on the real part of density matrix. This is also true in DFT when a pure exchangecorrelation (XC) functional is used. It can be seen from Eqs. (4) and (8) that $P_{n}$ is purely imaginary when $n$ is even and is real when $n$ is odd. When $n$ is even, $h^{(1)}$ is zero and the action of $L$ operator on $P_{n}$ thus only involves step (4).

From the above analysis, to achieve linear scaling in both CPU time and memory usage in the calculation of the absorption spectra and dynamics polarizability for very large 
TABLE I. CPU time for each step in TD-DFTB calculation in real time domain (unit: s) with a $10 \AA$ cutoff for first-order density matrix and without any cutoff.

\begin{tabular}{|c|c|c|c|c|c|c|c|c|}
\hline & \multicolumn{4}{|c|}{$10 \AA$ cutoff length } & \multicolumn{4}{|c|}{ Without any cutoff } \\
\hline & Step 1 & Step 2 & Step 3 & Step 4 & Step 1 & Step 2 & Step 3 & Step 4 \\
\hline$\left(\mathrm{H}_{2} \mathrm{O}\right)_{216}$ & 0.359 & 0.004 & 0.284 & 1.95 & 0.678 & 0.004 & 0.292 & 2.863 \\
\hline$\left(\mathrm{H}_{2} \mathrm{O}\right)_{432}$ & 1.148 & 0.011 & 1.052 & 6.882 & 3.528 & 0.012 & 1.086 & 13.958 \\
\hline$\left(\mathrm{H}_{2} \mathrm{O}\right)_{648}$ & 2.066 & 0.021 & 2.131 & 12.453 & 7.885 & 0.021 & 2.070 & 32.692 \\
\hline$\left(\mathrm{H}_{2} \mathrm{O}\right)_{864}$ & 3.131 & 0.031 & 4.135 & 19.491 & 15.125 & 0.031 & 4.144 & 60.961 \\
\hline
\end{tabular}

systems, sparse matrix multiplication as well as a cutoff for $P^{(1)}$ must be adopted. In our implementation, the sparse matrices are stored in a compressed sparse row format ${ }^{34}$ to save the nonzero elements only, and the SPARSEKIT (Ref. 35) is employed for sparse matrix multiplication. A more efficient sparse matrix multiplication scheme is possible by using multiatom blocks as recommended in Ref. 36. As for the cutoff for $P^{(1)}, P_{a b}^{(1)}$ in the orthogonal basis is set to zero if the distance between the centers of orbitals $a$ and $b$ is larger than a cutoff length $l_{1}$. In the calculation of the multiplication between $\mathrm{h}_{0}$ and $P^{(1)}$ with SPARSEKIT, we find that if the total nonzero number of $P^{(1)}$ is larger than half of the total elements of $P^{(1)}$, it is even slower than treating $P^{(1)}$ as a dense matrix. This means that higher efficiency can only be achieved for a relatively small $l_{1}$ with the present implementation. It is worth noting that the only approximation in the time-domain method presented here is the cutoff for $P^{(1)}$ compared with the tradition method based on Eq. (1). The error of the absorption spectra obtained with the present time-domain method without any cutoff for $P^{(1)}$ is only governed by the total propagation time. On the other hand, reasonable estimates for absorption spectra were achieved in our previous studies ${ }^{14}$ with a proper cutoff for $P^{(1)}$.

To test the efficiency and accuracy of our code, we calculate three-dimensional water clusters: $\left(\mathrm{H}_{2} \mathrm{O}\right)_{216},\left(\mathrm{H}_{2} \mathrm{O}\right)_{432}$, $\left(\mathrm{H}_{2} \mathrm{O}\right)_{648}$, and $\left(\mathrm{H}_{2} \mathrm{O}\right)_{864}$. The structures of these clusters are generated with HYPERCHEM V7.5. The water molecules are randomly distributed in a cubic box of lengths 18.7, 23.5, 27.0, and 29.6 $\AA$ with an average distance of $2.3 \AA$. All the calculations are performed on a single P5 $2.8 \mathrm{GHz}$ processor with 3 Gbyte memory. The percentages of nonzero ground state density matrix elements for these systems are $35 \%$, $22 \%, 16 \%$, and $13 \%$, respectively. The number of nonzero ground state Fock matrix is even smaller. With a $10 \AA$ cutoff for $P^{(1)}$, the percentages of nonzero first-order density matrix elements for these systems are 31\%, 20\%, 14\%, and $11 \%$. The CPU times for each step in the calculation of water clusters are listed in Table I. If no cutoff is adopted, the construction of $h^{(1)}$ is much faster than the matrix operations and step (3) is faster than step (1). For the cutoff case, step (1) can be even faster than step (3) for large systems. In addition, the matrix transformation is also faster than the calculation of the commutators. This is because the transformation matrix is a triangle matrix and very sparse. It can be seen from Table I that high efficiency is retained for the cutoff of the density matrix. The total CPU time for a propa- gation of 1 fs is shown in Fig. 1 for the calculations on these water clusters. It can also be seen that the total CPU time scales as $O\left(N^{2}\right)$ with the system size if no cutoff is applied, while it scales linearly with a $10 \AA$ cutoff for the first-order density matrix. The calculated absorption spectra of $\left(\mathrm{H}_{2} \mathrm{O}\right)_{864}$ due to an external field polarized along the $x$ direction are plotted in Fig. 2. It can be seen from Fig. 2 that the error caused by the $10 \AA$ cutoff for $P^{(1)}$ is negligible. This is because the excitations of the system are mostly localized on a single water molecule and are affected only by nearby water molecules. A small cutoff length for $P^{(1)}$ can thus result in accurate absorption spectra. Based on this, we conclude that the detailed coordinates of the water molecule in the cluster affect marginally the absorption spectra. In addition, with the present implementation, the total CPU time of a 35 fs propagation for $\left(\mathrm{H}_{2} \mathrm{O}\right)_{864}$ is about $16 \mathrm{~h}$ with the cutoff of $10 \AA$ and $55 \mathrm{~h}$ without any cutoff. This shows that with the present implementation we are able to calculate the absorption spectra and polarizabilty of very large three-dimensional (3D) systems. Note that the absorption spectra shown in Fig. 2 are only for the demonstration of the error due to the cutoff for $P^{(1)}$. The absorption spectra of water cluster are poorly described with TD-DFTB since Rydberg type excitations have dominant contribution to the absorption spectra for water and

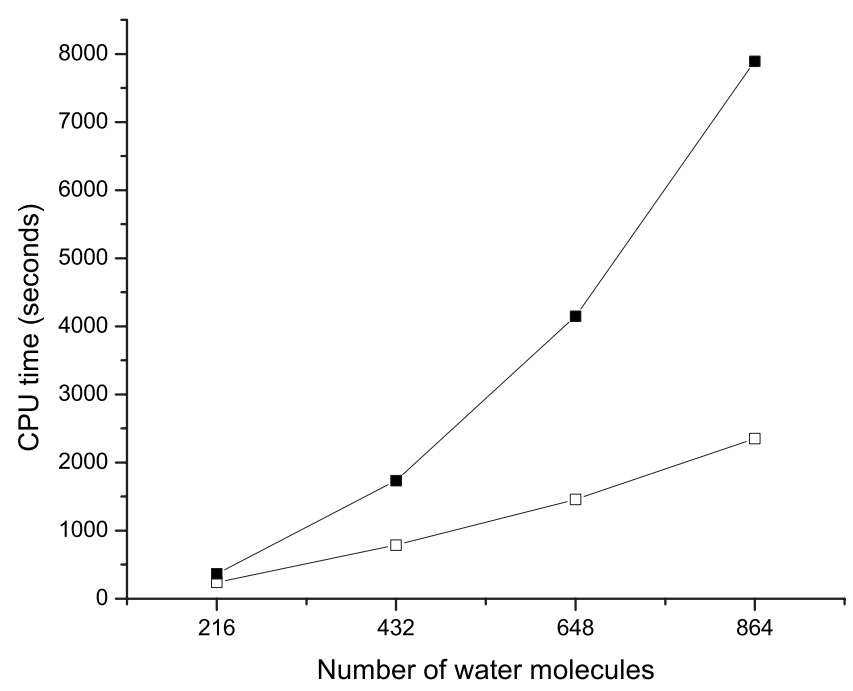

FIG. 1. The total CPU time for water cluster systems using TD-DFTB in real time domain with a propagation time of $1 \mathrm{fs}$ (empty square, with a $10 \AA$ cutoff for the first-order density matrix; filled square, without cutoff for the first-order density matrix). 


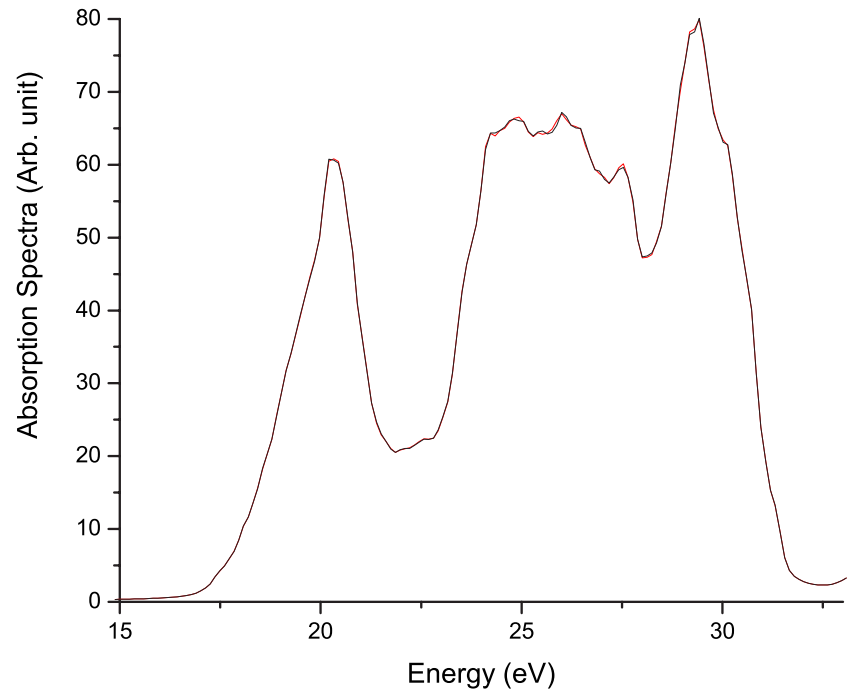

FIG. 2. (Color online) The absorption spectra of $\left(\mathrm{H}_{2} \mathrm{O}\right)_{864}$ calculated with TD-DFTB in real time domain. (black line, results without cutoff; red line, results with a $10 \AA$ cutoff).

diffuse basis functions as well as XC potentials with correct asymptotic behavior are required to obtain reasonable estimates for Rydberg type excitations. ${ }^{37}$

On the other hand, for delocalized excitations, which are usually results of the periodic structure of the corresponding systems, a larger cutoff length needs to be adopted to afford accurate estimates for the absorption spectra. These systems are preferably treated with a periodic boundary condition in the calculation of the absorption spectra. For those systems whose structures are not so regular, the excitations are much more localized and the method proposed here with a relatively short cutoff length for density matrix is expected to result in accurately calculated absorption spectra.

\section{CONCLUSION}

In the present work, we propagate the density matrix within TD-DFTB in real time to calculate the absorption spectra for very large systems. The time-dependent first- order change of the density matrix is calculated with high efficiency and accuracy using Chebyshev's expansion and the absorption spectra can be obtained though the imaginary part of the Fourier transformation for the time-dependent first-order dipole moment. To achieve linear scaling, the sparsity of the involving matrices is utilized and a cutoff for the first-order change of density matrix is employed. The prefactor for the scaling law of this method corresponds to the number of terms needed to converge the expansion in Eq. (7), which is decided by the total propagation time and the largest excitation energy of the system. In usual calculations, a total propagation time of 35 which leads to an energy resolution of $0.1 \mathrm{eV}$ is accurate enough. In DFTB method, the largest excitation energy is usually less than $50 \mathrm{eV}$, that is around $75 \mathrm{fs}^{-1}$. This means that in a norm calculation, the prefactor for the scaling law of the present method is around 3000. In fact, a smaller propagation time is also possible for small systems ${ }^{15}$ when spectra analysis method such as the filter-diagonalization method ${ }^{38-40}$ is used, which could reduce this prefactor. In our implementation, a compressed sparse row format is used to store the nonzero elements of the involving matrices and the SPARSEKIT package is employed for the multiplications between sparse matrices. With this implementation, both the CPU time and the memory usage are made to scale linearly with the system size. This facilitates the application of the present implementation for the calculation of absorption spectra of very large systems.

The absorption spectra of 3D water cluster $\left(\mathrm{H}_{2} \mathrm{O}\right)_{864}$ are calculated with the present approach. The CPU time is demonstrated to scale almost linearly with the system size for water clusters if cutoff for first-order density matrix is employed. The calculated absorption spectra for $\left(\mathrm{H}_{2} \mathrm{O}\right)_{864}$ with a $10 \AA$ cutoff length for first-order density matrix agree very well with those of no cutoff case. The cutoff for first-order density matrix results in reliable results for localized excitations. From these calculations, it is shown that the method presented here is very efficient and capable of describing the absorption spectra for large 3D systems. Linear scaling both in CPU time and memory usage can be achieved through a cutoff for the first-order change of the density matrix. *ghc@everest.hku.hk

†rauenheim@bccms.uni-bremen.de

${ }^{1}$ E. K. U. Gross and W. Kohn, Adv. Quantum Chem. 21, 255 (1990)

${ }^{2}$ M. E. Casida, in Recent Advances in Density Functional Methods, edited by D. P. Chong (World Scientific, Singapore, 1995), Vol. 1, p. 155.

${ }^{3}$ R. Bauernschmitt and R. Ahlrichs, Chem. Phys. Lett. 256, 454 (1996).

${ }^{4}$ C. Jamorski, M. E. Casida, and D. R. Salahub, J. Chem. Phys. 104, 5134 (1996).

${ }^{5}$ R. E. Stratmenn, G. E. Scuseria, and M. J. Frischer, J. Chem. Phys. 109, 8218 (1998).

${ }^{6}$ D. J. Tozer and N. C. Handy, J. Chem. Phys. 109, 10180 (1998).
${ }^{7}$ A. Görling, H. H. Heinze, S. Ph. Ruzankin, M. Staufer, and N. Rösch, J. Chem. Phys. 110, 2785 (1999).

${ }^{8}$ S. J. A. van Gisbergen, J. G. Snijders, and E. J. Baerends, Comput. Phys. Commun. 118, 119 (1999).

${ }^{9}$ S. Hirata and M. Head-Gordon, Chem. Phys. Lett. 314, 291 (1999).

${ }^{10}$ P. Salek, O. Vahtras, T. Helgaker, and H. Ågren, J. Chem. Phys. 117, 9630 (2002).

${ }^{11}$ Z. Rinkevicius, I. Tunell, P. Salek, O. Vahtras, and H. Ågren, J. Chem. Phys. 119, 34 (2003).

${ }^{12}$ E. R. Davidson, J. Comput. Phys. 17, 87 (1975).

${ }^{13}$ G. E. Scuseria, J. Phys. Chem. A 103, 4782 (1999)

${ }^{14}$ C. Y. Yam, S. Yokojima, and G. H. Chen, Phys. Rev. B 68, 153105 (2003); C. Y. Yam, S. Yokojima, and G. H. Chen, J. 
Chem. Phys. 119, 8794 (2003); W. Z. Liang, S. Yokojima, M. F. Ng, G. H. Chen, and G. He, J. Am. Chem. Soc. 123, 9830 (2001); W. Z. Liang, S. Yokojima, D. H. Zhou, and G. H. Chen, J. Phys. Chem. A 104, 2445 (2000); W. Z. Liang, S. Yokojima, and G. H. Chen, J. Chem. Phys. 110, 1844 (1999).

${ }^{15}$ F. Wang, C. Y. Yam, and G. Chen, J. Chem. Phys. 126, 134104 (2007)

${ }^{16}$ R. Baer and D. Neuhauser, J. Chem. Phys. 121, 9803 (2004).

${ }^{17}$ R. Kosloff, J. Phys. Chem. 92, 2087 (1988).

${ }^{18}$ G. Seifert, D. Porezag, and T. Frauenheim, Int. J. Quantum Chem. 58, 185 (1996).

${ }^{19}$ M. Elstner, D. Porezag, G. Jungnickel, J. Elsner, M. Haugk, T. Frauenheim, S. Suhai, and G. Seifert, Phys. Rev. B 58, 7260 (1998)

${ }^{20}$ T. Krüger, M. Elstner, P. Schiffels, and T. Frauenheim, J. Chem. Phys. 122, 114110 (2005).

${ }^{21}$ G. Zheng, S. Irle, and K. Morokuma, Chem. Phys. Lett. 412, 210 (2005).

${ }^{22}$ T. A. Niehaus, S. Suhai, F. Della Sala, P. Lugli, M. Elstner, G. Seifert, and T. Frauenheim, Phys. Rev. B 63, 085108 (2001).

${ }^{23}$ D. Heringer, T. A. Niehaus, M. Wanko, and T. Frauenheim, J. Comput. Chem. (to be published).

${ }^{24}$ B. Torralva, T. A. Niehaus, M. Elstner, S. Suhai T. Frauenheim, and R. E. Allen, Phys. Rev. B 64, 153105 (2001).

${ }^{25}$ T. A. Niehaus, D. Heringer, B. Torralva, and T. Frauenheim, Eur. Phys. J. D 35, 467 (2005).

${ }^{26}$ J. C. Slater, Quantum Theory of Molecules and Solids (McGraw-
Hill, New York, 1974), Vol. 4.

${ }^{27}$ S. H. Vosko, L. Wilk, and M. Nusair, Can. J. Phys. 58, 1231200 (1980).

${ }^{28}$ J. Fabian, L. A. Diaz, G. Seifert, and T. A. Niehaus, J. Mol. Struct.: THEOCHEM 594, 41 (2002).

${ }^{29}$ M. Wanko, M. Garavelli, F. Bernardi, T. A. Niehaus, T. Frauenheim, and M. Elstner, J. Chem. Phys. 120, 1674 (2004).

${ }^{30}$ S. Goedeck, Rev. Mod. Phys. 71, 1085 (1999).

${ }^{31}$ S. Yokojima and G. H. Chen, Phys. Rev. B 59, 7259 (1999).

${ }^{32}$ S. Yokojima and G. H. Chen, Chem. Phys. Lett. 292, 379 (1998).

${ }^{33}$ W. H. Press, B. P. Flannery, S. A. Teukolsky, and W. T. Vetterling, Numerical Recipes (Cambridge University Press, Cambridge, 1986).

${ }^{34}$ Y. Saad, Iterative Methods for Sparse Linear Systems (PWS, Boston, MA, 1996).

${ }^{35}$ Y. Saad, http://www.cs.umn.edu/Research/arpa/SPARSKIT/ sparskit.htm

${ }^{36}$ C. Saravana, Y. Shao, R. Baer, P. N. Ross, and M. Head-Gordon, J. Comput. Chem. 24, 618 (2003).

${ }^{37}$ M. E. Casida, C. Jamorski, K. C. Casida, D. R. Salahub, J. Chem. Phys. 108, 4439 (1998).

${ }^{38}$ M. R. Wall and D. Neuhauser, J. Chem. Phys. 102, 8011 (1995).

${ }^{39}$ V. A. Mandelshtam and H. S. Taylor, J. Chem. Phys. 106, 5085 (1997).

${ }^{40}$ J. W. Pang, T. Dieckman, J. Feigon, and D. Neuhauser, J. Chem. Phys. 108, 8360 (1998). 